EASD

Procedia
EURODYN 2020

XI International Conference on Structural Dynamics

M. Papadrakakis, M. Fragiadakis, C. Papadimitriou (eds.)

\title{
MODEL REDUCTION FOR STRUCTURES SUBJECTED TO BLAST LOADING BY USE OF DYNAMIC SUBSTRUCTURING
}

\author{
Linus Andersson, Peter Persson, Kent Persson \\ Department of Construction Sciences, Lund University \\ P.O. Box 118, SE-221 00 Lund, Sweden \\ e-mail: \{linus.andersson, peter.persson, kent.persson\}@construction.lth.se
}

Keywords: Dynamic substructuring, Blast loading, Direct time-integration, Beam frame structure

\begin{abstract}
In the present study, strategies are developed to enable time-efficient models for structures subjected to blast loading, appropriate for use in a structural design process. Dynamic substructuring is employed to obtain reduced models with localized nonlinearities, such as predefined plastic hinges in a beam-column structure. The parts of the substructures that remains linear elastic are modeled by Ritz-vectors whereas parts with a nonlinear response are retained as physical degrees-of-freedom. Furthermore, a time-stepping method is presented that is shown to be suitable for reduced models including local and predefined rigid-plastic behavior. The proposed methodology is applied and demonstrated in a numerical example of a concrete frame structure. Both the well-established Craig-Bampton method and reduction bases enriched by so-called correction modes are evaluated. For the load case studied, it is shown that the standard Craig-Bampton technique is suitable for reducing the substructures. Furthermore, it is shown that only a few Ritz-vectors are needed to sufficiently describe the deformation of the structure. However, additional modes are needed to ensure an accurate representation of the interface forces between the substructures.
\end{abstract}

ISSN:2311-9020 @ 2020 The Authors. Published by EASD Procedia.

Peer-review under responsibility of the Organizing Committee of EURODYN 2020.

doi: 10.47964/1120.9207.19160 


\section{INTRODUCTION}

Simplified models, such as equivalent single-degree-of-freedom (SDOF) systems, are often used for design of concrete members subjected to blast loading, an approach proposed already in the mid-1960s [1]. When this simplified approach is used for studying individual structural members, the supporting global structure is often considered as rigid and thus represented by prescribed displacement boundary conditions in the local response analyses. Hence, if a global response analysis is required it is in general performed in a subsequent stage, where the reaction forces computed in the local analyses are applied on the global structure. Such a procedure can be suitable if the stiffness and/or mass of the global structure are relatively large. However, in cases where the structural member is stiff and heavy, or even integrated as a part of the global lateral load-bearing structure, it can be necessary to employ a model of the coupled system. Furthermore, it can be necessary to include higher order modes to accurately capture the force transmitted between individual members and the global structure, in particular if the structure is subjected to a pulse with short duration and large peak pressure, as e.g. discussed in [2].

The dynamic response of a structure can e.g. be computed by use of a nonlinear finite element (FE) model, including the members subjected to external loading and the supporting global structure. However, the design of concrete structures subjected to blast loading is often an iterative process, where the cross-section dimensions and the amount and arrangement of bending reinforcement affect the dynamic response. Therefore, simplified and computationally efficient models are key in the conceptual design phase.

In the present study, strategies are developed to enable time-efficient models for structures subjected to blast loading, appropriate for use in a structural design process. More specifically, reduced models with localized nonlinearities, such as predefined plastic hinges in a beam-column structure, are obtained by use of dynamic substructuring (DS) (for an overview of DS techniques see e.g. $[3,4]$ ). Hence, parts that remains linear elastic are reduced and modeled by Ritz-vectors whereas the degrees-of-freedom (DOFs) included in parts with a nonlinear response are retained as physical DOFs. Furthermore, a time-stepping scheme is presented that is shown to be suitable for reduced models including local and predefined rigid-plastic behavior, such as frame structures with plastic hinges.

\section{SIMPLIFIED MODELING OF CONCRETE STRUCTURES SUBJECTED TO BLAST LOADING}

As for dynamic loading in general, the design of concrete structures subjected to blast loading can be challenging due to that the response is affected by the structure's mass, stiffness and strength. Hence, a modification of the dimensions, the amount and/or arrangement of reinforcement must, in general, be verified by an updated dynamic response analyses. Furthermore, a nonlinear response analysis is often required. The kinetic energy induced by the external pressure is converted into elastic and plastic strain energy, and if the plastic dissipation is omitted in the analysis the response can be inaccurate, e.g. resulting in a very conservative design. Therefore, simplified nonlinear SDOF models are often employed to evaluate the response of individual structural members.

In addition to the design of individual members, the global structure must be designed to resist progressive collapse and to ensure lateral stability. If a nonlinear response analysis is required for studying the global response, it is in general not straight-forward to construct a simplified model. Hence, a more refined nonlinear FE model of the whole structure can be necessary. 

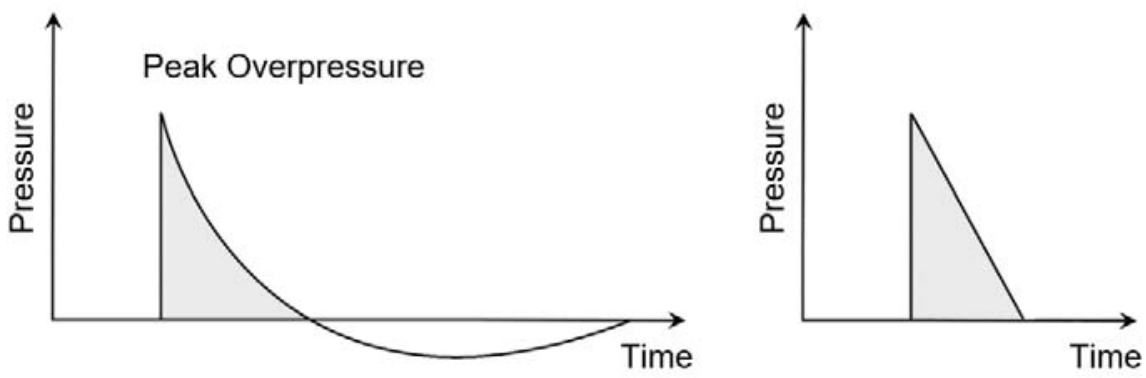

Figure 1: Typical (left) and idealized (right) blast pressure-time history.

\subsection{Impulse pressure due to unconfined explosion}

An unconfined explosion located on the ground surface results in a ground reflected shock wave that moves radially away from the center of the explosion [5]. If the explosion center is reasonable far from a structure, the pressure acting on the structure can be approximated by a uniform pressure. Upon impact, the initial wave is reinforced and reflected. The reflected impulse pressure acting on the structure is characterized by a very short duration and large peak pressure. For design purposes, the reflected impulse can, in general, be replaced by an equivalent triangular pulse, as shown in Figure 1. The actual duration is then replaced by a fictitious duration, computed from the peak reflected pressure and the reflected impulse.

\subsection{Reduced models for design of concrete members}

To ensure an adequate design of concrete members, such as beams and plates, subjected to blast loading, both the local response and the semi-global response (i.e. the global response of an individual member) must be considered. Hence, concrete members must be designed to resist local failure modes, such as punching shear failure, scabbing, spalling and penetration. Further, the shear force capacity and rotation capacity must be sufficiently large. Thus, it is crucial to ensure sufficient ductility and to avoid brittle failure modes.

A common procedure when evaluating the semi-global response of beams and plates is to set up an equivalent elasto-plastic SDOF system, where the yield force is derived from the ultimate moment capacity [1]. In fact, the yield force corresponds to the ultimate load, which implies that the maximum shear force in the dynamic response analysis is equal to the shear force given by a static yield line analysis. As e.g. shown in [2], the shear force computed using this simplified approach might be underestimated due to the neglection of higher order modes. Nonetheless, the approach can be reasonably accurate and in accordance with several design codes, such as UFC [5].

Using a simplified equivalent SDOF system implies that the position of the yield lines and/or plastic hinges can by assumed in accordance with a static yield line analysis, where the spatial load distribution correspond to the spatial distribution of the impulse pressure. This assumption is also the basis in the modeling strategies developed in Sections 3 and 4. However, it should be noted that this approach is primarily intended to be used in a conceptual design phase, i.e. a full nonlinear analysis can be necessary to verify the final design.

\section{DYNAMIC SUBSTRUCTURING OF STRUCTURES WITH PLASTIC HINGES}

By use of dynamic substructuring (DS) the aim is to efficiently compute the dynamic response of a structure by subdivision of the structure into substructures. Solving several substructures can be computationally less expensive than solving one large system. Moreover, DS can be employed to reduce systems with localized nonlinearities, such as predefined plastic 
hinges in a beam-column structure. The parts of the structure that remains linear elastic is then reduced and modeled by Ritz-vectors whereas the DOFs included in parts with a nonlinear response are retained as physical DOFs.

In most DS techniques reduction is performed on the substructure level. Hence, the substructure displacements $\mathbf{u}^{(s)}$ are represented by a reduced set of coordinates $\mathbf{q}^{(s)}$, given by

$$
\mathbf{u}^{(s)}=\mathbf{T q}^{(s)}
$$

where superscript $s$ is the substructure label and $\mathbf{T}$ is a $n^{(s)} \times m^{(s)}$ transformation matrix, whose columns are Ritz-vectors, or so-called component modes. Depending on the DS technique, the reduced vector $\mathbf{q}^{(s)}$ can include physical master (or boundary) DOFs and/or generalized coordinates. Typically, $m^{(s)}<<n^{(s)}$.

A FE formulation of a substructure leads to a linear equation of motion of the following form:

$$
\mathbf{M}^{(s)} \ddot{\mathbf{u}}^{(s)}+\mathbf{C}^{(s)} \dot{\mathbf{u}}^{(s)}+\mathbf{K}^{(s)} \mathbf{u}^{(s)}=\mathbf{p}^{(s)}
$$

where $\mathbf{p}^{(s)}$ is a $n^{(s)} \times 1$ substructure load vector and $\mathbf{M}^{(s)}, \mathbf{C}^{(s)}$ and $\mathbf{K}^{(s)}$ are the $n^{(s)} \times n^{(s)}$ substructure mass, damping and stiffness matrices, respectively. By inserting Equation (1) in (2) and pre-multiplying with $\mathbf{T}^{\mathrm{T}}$ the reduced subsystem is given by:

$$
\widetilde{\mathbf{M}}^{(s)} \ddot{\mathbf{q}}^{(s)}+\widetilde{\mathbf{C}}^{(s)} \dot{\mathbf{q}}^{(s)}+\widetilde{\mathbf{K}}^{(s)} \mathbf{q}^{(s)}=\widetilde{\mathbf{p}}^{(s)}
$$

where

$$
\widetilde{\mathbf{M}}^{(s)}=\mathbf{T}^{\mathrm{T}} \mathbf{M}^{(s)} \mathbf{T}, \quad \widetilde{\mathbf{C}}^{(s)}=\mathbf{T}^{\mathrm{T}} \mathbf{C}^{(s)} \mathbf{T}, \quad \widetilde{\mathbf{K}}^{(s)}=\mathbf{T}^{\mathrm{T}} \mathbf{K}^{(s)} \mathbf{T}, \quad \tilde{\mathbf{p}}^{(s)}=\mathbf{T}^{\mathrm{T}} \mathbf{p}^{(s)}
$$

The size of the reduced system matrices $\widetilde{\mathbf{M}}^{(s)}, \widetilde{\mathbf{C}}^{(s)}$ and $\widetilde{\mathbf{K}}^{(s)}$ is thus $m^{(s)} \times m^{(s)}$ and the reduced load vector $\tilde{\mathbf{p}}^{(s)}$ is $m^{(s)} \times 1$ (to simplify the notation the superscript $s$ will be left out until Section 3.3, on substructure coupling procedures). The substructures are then reassembled to form a reduced model of the full coupled system, why DS is sometimes referred to as Component Mode Synthesis (CMS).

Arguably the most straight-forward methods within DS are the so-called condensation methods, where only physical master DOFs are included in q. The most common condensation method is Guyan reduction, also referred to as static condensation due to that inertia effects are ignored [6]. The Guyan transformation matrix is given by

$$
\left[\begin{array}{l}
\mathbf{u}_{i} \\
\mathbf{u}_{b}
\end{array}\right]=\left[\begin{array}{c}
-\mathbf{K}_{i i}{ }^{-1} \mathbf{K}_{i b} \\
\mathbf{I}_{b b}
\end{array}\right] \mathbf{u}_{b}=\left[\begin{array}{c}
\boldsymbol{\Psi}_{i b} \\
\mathbf{I}_{b b}
\end{array}\right] \mathbf{u}_{b}=\mathbf{T}_{\mathrm{G}} \mathbf{q}
$$

where the subscripts $i$ and $b$ denotes the interior and interface boundary DOFs, $\mathbf{T}_{\mathrm{G}}$ is the Guyan transformation matrix and $\mathbf{q}$ contains the physical boundary DOFs. The columns of the transformation matrix are the so-called constraint modes, obtained by prescribing a unit displacement for a boundary DOF, while the interior DOFs are force-free and the other boundary DOFs are held fixed. Using this approach, "exact" results are achieved for static loading. However, due to neglected inertia effects the accuracy can be expected to decrease with an increasing forcing frequency.

Using a similar procedure but replacing the stiffness matrix with the dynamic stiffness matrix, exact result can instead be achieved for a certain forcing frequency, often referred to as dynamic reduction. There are also other more sophisticated condensation methods, such as Improved Reduction System (IRS) and System Equivalent Reduction Expansion Process (SEREP), 
where the number of exact resonances is less or equal to the number of boundary DOFs [7]. However, using condensation methods, it is not possible to both obtain a reduced model that is "statically complete" (i.e. a basis for all possible deformations that result from loading at the substructure boundary nodes) and at the same time keeping exact resonances. To achieve this, a CMS approach is required that uses generalized coordinates representing the amplitudes of additional Ritz-vectors.

Several CMS techniques have been developed since the 1960s, which can be divided into fixed-interface and free-interface methods. The most popular approach, a fixed-interface method, is CMS by Craig-Bampton (C-B), developed in the late 1960s [8]. The C-B method combines fixed-interface normal modes with so-called constraint modes, see further Section 3.1. Free-interface methods, using so-called free-interface component modes, were developed by MacNeal, Rubin and Craig and Chang in the 1970s [9,10,11]. In addition, another free-interface method, the dual Craig-Bampton method, was proposed in the early 2000s which, in contrast to the other free-interface methods, preserves the sparsity of the system matrices [12].

Reducing a structure with plastic hinges, it is important to employ a DS technique where the physical boundary DOFs, namely the rotational DOFs at the plastic hinges, are retained. Therefore, the CMS method proposed by Craig and Chang is excluded due to an assembling procedure that removes the physical boundary DOFs. The dual C-B method is an interesting approach since the interface forces are kept as DOFs in the assembled system. However, this method produces negative eigenvalues which make a stable time integration impossible [13] (an approach to overcome this issue is e.g. proposed in [13], unfortunately this approach includes removing all physical DOFs).

Apart from retaining the physical boundary DOFs at the plastic hinges, the plastic deformations of the assembled system must be resolved. Hence, the reduced subsystems should be "statically complete" and rigid body modes of the subsystems should be included in the reduction basis. Using the C-B method, the rigid body modes are not explicitly included in the reduction basis. However, the rigid body modes are spanned by the constraint modes. In fact, the set of constraint modes is a basis for all possible deformations that result from loading at the substructure boundary nodes [14]. In addition to the C-B method, the free-interface methods by MacNeal and Rubin retains the physical boundary DOFs in the assembled system and explicitly includes the rigid body modes in the reduction basis.

In summary, either the fixed-interface C-B method or the free-interface methods by MacNeal and Rubin are judged to be the most suitable methods for reducing a beam-column structure including predefined plastic joints. A free-interface approach can be expected to be more accurate upon yielding, i.e. when the joint stiffness is zero, whereas a fixed-interface approach can be expected to be more accurate when the joints are fixed, i.e. when yielding does not occur. Based on this observation it is assumed that the classic C-B approach, which preserves the sparsity of the system matrices, is the preferred choice.

\subsection{Craig-Bampton method}

The Craig-Bampton method combines the retained physical DOFs with generalized coordinates corresponding to the amplitudes of so-called fixed-interface normal modes. Neglecting damping the partitioned mass and stiffness matrices can be written as:

$$
\left[\begin{array}{ll}
\mathbf{M}_{i i} & \mathbf{M}_{i b} \\
\mathbf{M}_{b i} & \mathbf{M}_{b b}
\end{array}\right]\left[\begin{array}{l}
\ddot{\mathbf{u}}_{i} \\
\ddot{\mathbf{u}}_{b}
\end{array}\right]+\left[\begin{array}{cc}
\mathbf{K}_{i i} & \mathbf{K}_{i b} \\
\mathbf{K}_{b i} & \mathbf{K}_{b b}
\end{array}\right]\left[\begin{array}{c}
\mathbf{u}_{i} \\
\mathbf{u}_{b}
\end{array}\right]=\left[\begin{array}{l}
\mathbf{p}_{i} \\
\mathbf{p}_{b}
\end{array}\right]
$$

By setting the boundary displacements to zero in Equation (6), the fixed-interface normal modes are obtained by the generalized eigenvalue problem: 


$$
\left(\mathbf{K}_{i i}-\omega_{j}^{2} \mathbf{M}_{i i}\right)\left\{\phi_{i}\right\}_{j}=0
$$

where $\mathbf{K}_{i i}$ and $\mathbf{M}_{i i}$ are the interior stiffness and mass matrices. The eigenvectors are normalized so that $\boldsymbol{\Phi}_{i i}{ }^{\mathrm{T}} \mathbf{M}_{i i} \boldsymbol{\Phi}_{i i}=\mathbf{I}_{i i}$, where $\boldsymbol{\Phi}_{i i}$ is the complete set of fixed-interface normal modes. The physical coordinates can then be represented as:

$$
\left[\begin{array}{l}
\mathbf{u}_{i} \\
\mathbf{u}_{b}
\end{array}\right]=\left[\begin{array}{ll}
\mathbf{\Phi}_{i k} & \boldsymbol{\Psi}_{i b} \\
\mathbf{0}_{b k} & \mathbf{I}_{b b}
\end{array}\right]\left[\begin{array}{l}
\mathbf{q}_{k} \\
\mathbf{u}_{b}
\end{array}\right]=\mathbf{T}_{\mathrm{C}-\mathrm{B}} \mathbf{q}
$$

where the subscript $k$ denotes the kept fixed-interface normal modes, $\mathbf{T}_{\mathrm{C}-\mathrm{B}}$ is the CraigBampton transformation matrix, $\mathbf{q}_{k}$ is the generalized coordinates and $\left[\begin{array}{ll}\boldsymbol{\Psi}_{i b} & \mathbf{I}_{b b}\end{array}\right]^{\mathrm{T}}$ is the constraint mode matrix, equivalent to the Guyan reduction basis. Hence, the Craig-Bampton method can be interpreted as an extension of the Guyan reduction where the neglected inertia terms are compensated by including a set of fixed-interface normal modes.

\subsection{Craig-Bampton with Modal Truncation Augmentation}

The fixed-interface normal modes included in the $\mathrm{C}-\mathrm{B}$ reduction basis can be augmented by so-called high order static correction modes, which can be interpreted as a form of generalization of the Guyan static modes. The C-B method with so-called Modal Truncation Augmentation (MTA) is presented in [15].

If the internal forces are zero, the top row of Equation (6) can be rewritten as:

$$
\mathbf{M}_{i i} \ddot{\mathbf{u}}_{i}+\mathbf{K}_{i i} \mathbf{u}_{i}=-\mathbf{M}_{i b} \ddot{\mathbf{u}}_{b}-\mathbf{K}_{i b} \mathbf{u}_{b}
$$

Hence, the substructure can be considered excited by imposed displacements on its boundary. Further, the internal displacements can be approximated as

$$
\mathbf{u}_{i}=\mathbf{u}_{i, \text { stat }}+\mathbf{y}
$$

where $\mathbf{u}_{i, \text { stat }}=-\mathbf{K}_{i i}{ }^{-1} \mathbf{K}_{i b} \mathbf{u}_{b}$ is the quasi-static solution modified by the second term $\mathbf{y}$ to obtain the dynamic response, i.e. similarly to the well-established mode acceleration method, e.g. described in [16]. By inserting Equation (10) into Equation (9) the dynamic response of the relative solution is given by

$$
\mathbf{M}_{i i} \ddot{\mathbf{y}}+\mathbf{K}_{i i} \mathbf{y}=-\mathbf{M}_{i i} \ddot{\mathbf{u}}_{i, \text { stat }}-\mathbf{M}_{i b} \ddot{\mathbf{u}}_{b}=\mathbf{Y} \ddot{\mathbf{u}}_{b}
$$

where $\mathbf{Y}=\mathbf{M}_{i i} \mathbf{K}_{i i}{ }^{-1} \mathbf{K}_{i b}-\mathbf{M}_{i b}$, which can be interpreted as inertia forces associated to static modes [15]. This procedure can be continued by replacing $\mathbf{y}$ with a quasi-static solution and a dynamic correction. Hence, a recursive procedure is obtained, indicating that the dynamic response can be approximated as

$$
\mathbf{u}_{i} \approx-\mathbf{K}_{i i}{ }^{-1} \mathbf{K}_{i b} \mathbf{u}_{b}+\sum_{j=1}^{n}\left(-\mathbf{K}_{i i}{ }^{-1} \mathbf{M}_{i i}\right)^{j-1} \mathbf{K}_{i i}{ }^{-1} \mathbf{Y} \frac{\mathrm{d}^{2 j} \mathbf{u}_{b}}{\mathrm{~d} t^{2 j}}
$$

where $n$ is the number of static corrections.

According to Equation (12) a dynamic response analysis is not included in the approximation. However, if a dynamic analysis including $k$ eigenmodes has been performed, part of the corrections is already considered. The expression for the higher order corrections can then be rewritten as: 


$$
\mathbf{u}_{i, c o r . j} \approx\left(\mathbf{K}_{i i}{ }^{-1}-\sum_{r=1}^{k} \frac{\phi_{r} \phi_{r}{ }^{\mathrm{T}}}{\omega_{r}^{2}}\right)\left(-\mathbf{M}_{i i} \mathbf{K}_{i i}{ }^{-1}\right)^{j-1} \mathbf{Y} \frac{\mathrm{d}^{2 j} \mathbf{u}_{b}}{\mathrm{~d} t^{2 j}}
$$

where we use that the inverse of the stiffness matrix can be expressed as

$$
\mathbf{K}_{i i}{ }^{-1}=\sum_{r=1}^{N} \frac{\phi_{r} \boldsymbol{\phi}_{r}^{\mathrm{T}}}{\omega_{r}^{2}}=\sum_{r=1}^{k} \frac{\boldsymbol{\phi}_{r} \boldsymbol{\phi}_{r}^{\mathrm{T}}}{\omega_{r}^{2}}+\sum_{r=k+1}^{N} \frac{\boldsymbol{\phi}_{r} \boldsymbol{\phi}_{r}^{\mathrm{T}}}{\omega_{r}^{2}}
$$

where $N$ is the total number of eigenmodes, and $\phi_{r}$ and $\omega_{r}$ is the eigenmode and eigenfrequency for mode $r$. Hence, the space already spanned by the fixed-interface normal modes is removed from the corrections. The total response is then given by

$$
\mathbf{u}_{i} \approx \sum_{r=1}^{k} \phi_{r} \mathrm{q}_{r}+\sum_{j=0}^{n} \mathbf{u}_{i, c o r . j}
$$

where $\mathbf{u}_{i, \text { cor } .0}=-\mathbf{K}_{i i}{ }^{-1} \mathbf{K}_{i b} \mathbf{u}_{b}$. Furthermore, the amplitudes $\frac{\mathrm{d}^{2 j} \mathbf{u}_{b}}{\mathrm{~d} t^{2 j}}$ can be treated as separate DOFs, i.e. instead of high order corrections the modal basis is augmented by high order correction modes, also referred to as force-dependent Ritz-vectors or Krylov vectors. Hence, the $j$-th order correction modes is given by:

$$
\mathbf{x}_{i, c o r . j}=\left(\mathbf{K}_{i i}{ }^{-1}-\sum_{r=1}^{k} \frac{\phi_{r} \boldsymbol{\phi}_{r}{ }^{\mathrm{T}}}{\omega_{r}^{2}}\right)\left(\mathbf{M}_{i i} \mathbf{K}_{i i}{ }^{-1}\right)^{j-1} \mathbf{Y}
$$

Note that the number of vectors generated in each iteration equals the number of boundary DOFs, why the subspace spanned by the generated vectors is sometimes referred to as a blockKrylov subspace [14]. The static correction modes are both mass- and stiffness-orthogonal to the fixed-interface normal modes. However, they are not mutually orthogonal. This can e.g. be achieved by solving a small eigenvalue problem:

$$
\left(\mathbf{X}_{c o r}^{\mathrm{T}} \mathbf{K} \mathbf{X}_{c o r}\right) \mathbf{Z}=\left(\boldsymbol{X}_{c o r}^{\mathrm{T}} \mathbf{M} \mathbf{X}_{c o r}\right) \mathbf{Z} \mathbf{\Lambda}
$$

where $\mathbf{X}_{\text {cor }}$ is the correction mode matrix. The eigenvectors are normalized so that $\mathbf{Z}^{\mathrm{T}}\left(\mathbf{X}_{\text {cor }}^{\mathrm{T}} \mathbf{M} \mathbf{X}_{\text {cor }}\right) \mathbf{Z}=\mathbf{I}$ and the orthonormal basis of the correction vectors is then given by $\widetilde{\mathbf{X}}_{\text {cor }}=\mathbf{X}_{\text {cor }} \mathbf{Z}$. The relation between the substructure physical DOFs and the reduced coordinate is then given by

$$
\left[\begin{array}{c}
\mathbf{u}_{i} \\
\mathbf{u}_{b}
\end{array}\right]=\left[\begin{array}{ccc}
\boldsymbol{\Phi}_{i k} & \widetilde{\mathbf{X}}_{c o r} & \boldsymbol{\Psi}_{i b} \\
\mathbf{0}_{b k} & \mathbf{0}_{b n} & \mathbf{I}_{b b}
\end{array}\right]\left[\begin{array}{c}
\mathbf{q}_{k} \\
\mathbf{q}_{n} \\
\mathbf{u}_{b}
\end{array}\right]=\mathbf{T}_{\mathrm{C}-\mathrm{B}, \mathrm{cor}} \mathbf{q}
$$

where $\mathbf{q}_{n}$ is the amplitudes of the correction modes and $\mathbf{T}_{\mathrm{C}-\mathrm{B}, \mathrm{cor}}$ is the C-B transformation matrix augmented by correction modes.

Using the above procedure, correction modes are generated based on interface excitation. Using a similar procedure, the correction modes, or so-called force dependent Ritz-vectors, can also be generated based on external loads applied to the substructure interior DOFs. 


\subsection{Substructure coupling procedures}

Since the physical boundary DOFs are preserved in the C-B reduction process the assembly method is straight-forward, hence each substructure can be treated as a super-element. However, the substructures can either be assembled by eliminating one set of interface DOFs, a so-called primal assembled system, or so that all interface DOFs are preserved, a so-called dual assembled system. by

The block diagonal system matrices for a structure consisting of $N_{S}$ substructures are given

$$
\mathbf{M}=\operatorname{diag}\left(\mathbf{M}^{(s)}\right), \quad \mathbf{C}=\operatorname{diag}\left(\mathbf{C}^{(s)}\right), \quad \mathbf{K}=\operatorname{diag}\left(\mathbf{K}^{(s)}\right)
$$

where $\mathbf{M}, \mathbf{C}$ and $\mathbf{K}$ have size $n \times n$ and the superscript $s$ is the substructure label. The equation of motion can then be written as

$$
\mathbf{M u ̈}+\mathbf{C} \dot{\mathbf{u}}+\mathbf{K u}=\mathbf{p}
$$

where $\mathbf{u}$ is the $n \times 1$ global displacement vector, including all DOFs at the interfaces between substructures. Assuming compatible meshes, constraints enforced between substructures for individual DOF pairs, e.g. $\mathrm{u}_{i}^{\left(s_{1}\right)}=\mathrm{u}_{j}^{\left(s_{2}\right)}$, can be written in matrix form:

$$
\mathbf{B u}=\mathbf{0}
$$

where the constraint matrix $\mathbf{B}$ is a signed Boolean matrix with size $m \times n$, where $m$ is the number of constraints and $n$ is the total number of DOFs.

As e.g. shown in [3], a primal assembly can be enforced by using the so-called Boolean localization matrix $\mathbf{L}$, found by computing the null-space for $\mathbf{B}$, i.e.

$$
\mathbf{L}=\operatorname{Null}(\mathbf{B})
$$

The relation between the global displacement vector and a displacement vector with a unique set of interface DOFs, $\mathbf{u}_{P}$, is then given by

$$
\mathbf{u}=\mathbf{L} \mathbf{u}_{P}
$$

By inserting Equation (23) in Equation (20) and pre-multiplying with $\mathbf{L}^{\mathrm{T}}$ the equation of motion for the primal assembled system is given by

$$
\mathbf{M}_{P} \ddot{\mathbf{u}}_{P}+\mathbf{C}_{P} \dot{\mathbf{u}}_{P}+\mathbf{K}_{P} \mathbf{u}_{P}=\mathbf{p}_{P}
$$

where

$$
\mathbf{M}_{P}=\mathbf{L}^{\mathrm{T}} \mathbf{M L}, \quad \mathbf{C}_{P}=\mathbf{L}^{\mathrm{T}} \mathbf{C} \mathbf{L}, \quad \mathbf{K}_{P}=\mathbf{L}^{\mathrm{T}} \mathbf{K} \mathbf{L}, \quad \mathbf{p}_{P}(\mathrm{t})=\mathbf{L}^{\mathrm{T}} \mathbf{p}
$$

The number of DOFs in the primal assembled system is thus $n_{P}=n-m$ and, accordingly, the size of $\mathbf{M}_{P}, \mathbf{C}_{P}, \mathbf{K}_{P}$ is $\left[n_{P} \times n_{P}\right]$ and $\mathbf{p}_{P}$ is $\left[n_{P} \times 1\right]$.

Instead of eliminating one set of DOFs, a dual assembled system can be achieved by enforcing the interface constraints using Lagrange multipliers $\lambda$. The system of equations can then be written as 


$$
\left[\begin{array}{cc}
\mathbf{M} & \mathbf{0} \\
\mathbf{0} & \mathbf{0}
\end{array}\right]\left[\begin{array}{l}
\ddot{\mathbf{u}} \\
\lambda
\end{array}\right]+\left[\begin{array}{ll}
\mathbf{C} & \mathbf{0} \\
\mathbf{0} & \mathbf{0}
\end{array}\right]\left[\begin{array}{l}
\dot{\mathbf{u}} \\
\lambda
\end{array}\right]+\left[\begin{array}{cc}
\mathbf{K} & \mathbf{B}^{\mathrm{T}} \\
\mathbf{B} & \mathbf{0}
\end{array}\right]\left[\begin{array}{l}
\mathbf{u} \\
\boldsymbol{\lambda}
\end{array}\right]=\left[\begin{array}{l}
\mathbf{p} \\
\mathbf{0}
\end{array}\right]
$$

thus, preserving all interface DOFs [17]. Note that $\lambda$ can be interpreted as an interface force vector, i.e. the forces required to enforce equal displacements for interface DOF pairs.

A dual assembled system can also be enforced using the penalty method, e.g. expressed as

$$
\mathbf{M} \ddot{\mathbf{u}}+\mathbf{C} \dot{\mathbf{u}}+\left(\mathbf{K}+\alpha \mathbf{B}^{\mathrm{T}} \mathbf{B}\right) \mathbf{u}=\mathbf{p}
$$

where $\alpha$ is the penalty stiffness, chosen sufficiently large so that $\mathbf{B u} \approx \mathbf{0}$ [17]. Note that this approach corresponds to adding stiff springs between the constrained DOFs.

The preferred assembly method, or constraint enforcement method, depend on several factors such as the analysis type, the output data of interested, etc.

\section{TIME-STEPPING METHOD FOR STRUCTURES WITH RIGID-PERFECTLY PLASTIC JOINTS}

Simplified models are often used for design of concrete structures where linear elastic beam elements are combined with predefined discrete plastic hinges, approximated as rigid-perfectly plastic. To enforce a rigid-perfectly plastic coupling between two DOFs, namely the rotational DOFs at the plastic hinges, one alternative is to utilize the penalty method, i.e. by introducing a stiff elastic-perfectly plastic rotational spring. However, one obvious problem when using the penalty method is to choose a suitable penalty stiffness - if it is too low, the results will be inaccurate and if it is too large, the system equations will be ill-conditioned with respect to inversion. Furthermore, the highest eigenfrequency of the system can be expected to increase significantly, which can result in a very small critical time increment size in conditionally stable time integration schemes.

Another alternative is to set up a dual assembled system according to Equation (26) and setting a maximum value, corresponding to the plastic moment, for the Lagrange multipliers. The Lagrange multipliers will then be either known, i.e. treated as external forces equal to the plastic moments, or unknown. However, ensuring stability for a system including Lagrange multipliers, for so-called Differential-Algebraic Equations (DAEs), is often found to be problematic in direct time integration of dual assembled systems. For example, a standard Newmark time integration scheme, assuming constant average accelerations, is in this case unconditionally unstable. However, methods to ensure stability exist, see e.g. [18]. Nonetheless the available time integration schemes are somewhat limited.

Instead of using a pure dual or primal formulation, a methodology is proposed where the system is reassembled when yielding of the rigid-perfectly plastic hinges starts or stops. Such a procedure implies that the nonlinear analysis is performed as a series of linear analyses. Hence, the plastic hinges are either modeled as fixed by eliminating one of the rotational DOFs or as external moments applied on both rotational DOFs at the plastic hinges. This approach can be expected to be particularly suitable for systems consisting of reduced substructures, with a limited number of plastic hinges. The critical time increment is then related to the highest eigenfrequency of the assembled substructures, which in turn have already been reduced by a modal truncation on the substructure level, removing the high frequency content. Furthermore, high frequency noise induced by penalty elements is avoided. 


\subsection{Time-stepping algorithm}

Starting with the system in Equation (20), a $m \times n$ signed Boolean matrix B can be constructed to define all the interface couplings between the substructures. The couplings can either be fixed constraints or rigid-perfectly plastic couplings. The matrix is then partitioned as:

$$
\mathbf{B}=\left[\begin{array}{l}
\mathbf{B}_{r} \\
\mathbf{B}_{c} \\
\mathbf{B}_{y}
\end{array}\right]
$$

where $\mathbf{B}_{r}$ defines $m_{r}$ constraints (or boundary conditions) that are not limited by a yield force, e.g. the translational DOFs at a plastic hinge, $\mathbf{B}_{c}$ defines the $m_{c}$ rigid-plastic couplings not yielding and $\mathbf{B}_{y}$ defines the $m_{y}$ rigid-plastic couplings currently yielding. Hence, the Boolean matrices $\mathbf{B}_{c}$ and $\mathbf{B}_{y}$ must be updated in a time-stepping scheme. The constraints defined by $\mathbf{B}_{r}$ are enforced using a primal formulation before the time-stepping is initialized, hence by eliminating $m_{r}$ interface DOFs. The equation of motion for the assembled system can then be written as

$$
\widehat{\mathbf{M}} \ddot{\mathbf{u}}_{r}+\widehat{\mathbf{C}} \dot{\mathbf{u}}_{r}+\widehat{\mathbf{K}} \mathbf{u}_{r}=\hat{\mathbf{p}}
$$

where

$$
\widehat{\mathbf{M}}=\mathbf{L}_{r}^{\mathrm{T}} \mathbf{M L}_{r}, \quad \widehat{\mathbf{C}}=\mathbf{L}_{r}^{\mathrm{T}} \mathbf{C} \mathbf{L}_{r}, \quad \widehat{\mathbf{K}}=\mathbf{L}_{r}^{\mathrm{T}} \mathbf{K} \mathbf{L}_{r}, \quad \hat{\mathbf{p}}=\mathbf{L}_{r}^{\mathrm{T}} \mathbf{p}
$$

and where the Boolean localization matrix $\mathbf{L}_{r}=\operatorname{Null}\left(\mathbf{B}_{r}\right)$. To simplify the implementation the eliminated DOFs are then removed from the matrices $\mathbf{B}_{c}$ and $\mathbf{B}_{y}$, i.e. by removing the corresponding $m_{r}$ columns containing zeros. The size of the updated matrices $\widehat{\mathbf{B}}_{c}$ and $\widehat{\mathbf{B}}_{y}$ is then $m_{c} \times\left(n-m_{r}\right)$ and $m_{y} \times\left(n-m_{r}\right)$, respectively. At the initial stage, the couplings defined by $\widehat{\mathbf{B}}_{c}$ is enforced in the same manner by computing $\mathbf{L}_{c}=\operatorname{Null}\left(\widehat{\mathbf{B}}_{c}\right)$. At each time increment $t^{(i)}$, the interface forces $\lambda^{(i)}$ is computed from the expression

$$
\widehat{\mathbf{B}}_{c}^{\mathrm{T}} \lambda^{(i)}=\hat{\mathbf{p}}^{(i)}-\widehat{\mathbf{M}} \mathbf{L}_{c} \ddot{\mathbf{u}}_{c}{ }^{(i)}-\widehat{\mathbf{C}} \mathbf{L}_{c} \dot{\mathbf{u}}_{c}{ }^{(i)}-\widehat{\mathbf{K}} \mathbf{L}_{c} \mathbf{u}_{c}{ }^{(i)}
$$

where the relation $\mathbf{u}_{r}=\mathbf{L}_{c} \mathbf{u}_{c}$ has been applied. If the interface force for a coupling is larger than the specified yield force, the Boolean matrices $\widehat{\mathbf{B}}_{c}$ and $\widehat{\mathbf{B}}_{y}$ are updated, i.e. the row in $\widehat{\mathbf{B}}_{c}$ defining the coupling is moved to $\widehat{\mathbf{B}}_{y}$. Thereafter, the system is reassembled by computing the localization matrix $\mathbf{L}_{c}=\operatorname{Null}\left(\widehat{\mathbf{B}}_{c}\right)$ and applying it to Equation (29). The couplings currently yielding are then considered by adding the yield forces as external loads, hence

$$
\mathbf{L}_{c}^{\mathrm{T}} \widehat{\mathbf{M}} \mathbf{L}_{c} \ddot{\mathbf{u}}_{c}{ }^{(i)}+\mathbf{L}_{c}^{\mathrm{T}} \widehat{\mathbf{C}} \mathbf{L}_{c} \dot{\mathbf{u}}_{c}{ }^{(i)}+\mathbf{L}_{c}^{\mathrm{T}} \widehat{\mathbf{K}} \mathbf{L}_{c} \mathbf{u}_{c}{ }^{(i)}=\mathbf{L}_{c}^{\mathrm{T}}\left(\widehat{\mathbf{p}}^{(i)}-\widehat{\mathbf{B}}_{y}^{\mathrm{T}} \mathbf{f}_{y}\right)
$$

where $\mathbf{f}_{y}$ is a $m_{y} \times 1$ vector containing the specified yield forces. Unloading of the $m_{y}$ couplings currently yielding is detected by checking the relative velocity sign, i.e. if

$$
\operatorname{sign}\left(\left\{\widehat{\mathbf{B}}_{y} \dot{\mathbf{u}}_{r}{ }^{(i)}\right\}_{j}\right) \neq \operatorname{sign}\left(\left\{\widehat{\mathbf{B}}_{y} \dot{\mathbf{u}}_{r}{ }^{(i+1)}\right\}_{j}\right)
$$

where subscript $j$ denotes an element of the column vector $\widehat{\mathbf{B}}_{y} \dot{\mathbf{u}}_{r}$. If unloading occurs, the system is updated by moving rows from $\widehat{\mathbf{B}}_{y}$ to $\widehat{\mathbf{B}}_{c}$, computing the localization matrix $\mathbf{L}_{c}=\operatorname{Null}\left(\widehat{\mathbf{B}}_{c}\right)$ and applying it to Equation (29). 
The time integration between the system updates can be performed in a standard manner by the Newmark- $\beta$ method, as e.g. described in [19]. However, for a nonlinear system including both elastic and plastic deformations it is convenient to use incremental quantities,

$$
\Delta \mathbf{u}^{(i)}=\mathbf{u}^{(i+1)}-\mathbf{u}^{(i)}, \quad \Delta \mathbf{u}^{(i)}=\mathbf{u}^{(i+1)}-\mathbf{u}^{(i)}, \quad \Delta \ddot{\mathbf{u}^{(i)}}=\ddot{\mathbf{u}}^{(i+1)}-\ddot{\mathbf{u}}^{(i)}, \quad \Delta \mathbf{p}^{(i)}=\mathbf{p}^{(i+1)}-\mathbf{p}^{(i)}
$$

In fact, the time-stepping algorithm is constructed so that the computed incremental displacements are always transformed and appended to $\mathbf{u}_{r}$, thus

$$
\mathbf{u}_{r}{ }^{(i+1)}=\mathbf{u}_{r}{ }^{(i)}+\mathbf{L}_{c} \Delta \mathbf{u}_{c}{ }^{(i)}
$$

Consequently, the displacement vector need not to be transformed at the system updates. Merging DOF pairs in couplings where yielding is stopped would certainly be an ambiguous operation, since the displacement in each DOF in general will differ.

Upon yielding, the system is updated so that couplings where yielding is initiated are released. The interpretation of this update is straight-forward since the initial state of the physical quantities (i.e. displacements, velocities, accelerations) in the new configuration is well defined by the previous state. If yielding is stopped, rigid couplings are enforced by eliminating one set of interface DOFs. The velocities for the constrained DOFs are by definition equal when unloading is detected and it is thus straight-forward to eliminate one set of DOFs. The initial acceleration in the updated system is computed as

$$
\ddot{\mathbf{u}}_{c}{ }^{(i)}=\left(\mathbf{L}_{c}^{\mathrm{T}} \widehat{\mathbf{M}} \mathbf{L}_{c}\right)^{-1} \mathbf{L}_{c}^{\mathrm{T}}\left(\widehat{\mathbf{p}}^{(i)}-\widehat{\mathbf{B}}_{y}^{\mathrm{T}} \mathbf{f}_{y}-\widehat{\mathbf{C}} \dot{\mathbf{u}}_{r}{ }^{(i)}-\widehat{\mathbf{K}} \mathbf{u}_{r}{ }^{(i)}\right)
$$

Note that the time increment between system updates, $\Delta t^{(i)}=t^{(i)}-t^{(i-1)}$, is zero. Hence, the velocities and deformations are equal in increment $i$ and $i-1$. However, the accelerations are changed at the system updates in accordance with Equation (36). Even though an instantaneous change of the accelerations might seem unreasonable, this is a consequence of the unphysical assumption of a perfectly rigid connection, which however can be a very useful approximation; in particular when modeling plastic hinges in beam-column structures. Furthermore, it should be noted that both the strain and kinetic energy is preserved.

\subsection{Remarks on performance and accuracy}

In contrast to a conventional implicit direct time integration, the proposed time-stepping scheme, as presented above, is a non-iterative procedure. However, in general an adjustment of the last increment size before a system update is needed to ensure that the system is updated just before yielding start or stop. This adjustment can be set up in an iterative fashion.

If the system consists of reduced substructures the critical time increment, in a conditionally stable time integration scheme, can be expected to be reasonably large since the high frequency content has been removed in the reduction process on the substructure level. The time increment size prescribed in the analysis is then instead governed by the precision of the requested timehistories. Consequently, the Newmark parameters can be optimized to increase the analysis accuracy (or performance) rather than to ensure stability. For example, setting the parameters to $\beta=1 / 12$ and $\gamma=1 / 2$ ensures forth-order accuracy and $\beta=0$ and $\gamma=1 / 2$ enables the explicit central difference scheme [19]. Furthermore, if the system at hand is relatively small and if the number of system updates can be assumed rather limited, the critical time increment can be updated based on an eigenvalue analysis of the updated system. However, an alternative is to prescribe a conservative time increment and thereby skip this check. 


\section{NUMERICAL EXAMPLE: CONCRETE FRAME SUBJECTED TO BLAST LOADING}

A concrete frame structure subjected to blast loading is studied to demonstrate the discussed substructuring and time-stepping method. The dynamic response is computed using a simplified two-dimensional model, suitable in a conceptual design phase. Various strategies to reduce the model by use of DS are investigated, both in terms of accuracy and computationally efficiency (i.e. number of DOFs). Focus is primarily on evaluating DS techniques applied to the lateral load-bearing structure that is not subjected to external loading, i.e. the supporting structure consisting of the horizontal beam and the right column, as shown in Figure 2a. The dynamic response is computed using the time-stepping scheme proposed in Section 4. Furthermore, the highest eigenfrequency, which is related to the critical increment size in conditionally stable time-integration schemes, is evaluated for the reduced models.

The concrete frame is subjected to a uniformly distributed reflected impulse pressure of $1500 \mathrm{~Pa} \cdot \mathrm{s}$, as shown in Figure 2a. The pulse is approximated by an equivalent triangular pulse with a fictitious duration of $10 \mathrm{~ms}$ and a peak reflected pressure of $300 \mathrm{kPa}$ at $t=0 \mathrm{~s}$. The length of the frame members is $3 \mathrm{~m}$ and the cross-section width and height, for the columns as well as the horizontal beam, are $1000 \mathrm{~mm}$ and $200 \mathrm{~mm}$, respectively. Young's modulus for concrete is set to $32 \mathrm{GPa}$ and the density for reinforced concrete is set to $2500 \mathrm{~kg} / \mathrm{m}^{3}$. The ultimate moment capacity for the columns and the horizontal beam is set to $60 \mathrm{kNm}$.

\subsection{Frame structure model reduced by dynamic substructuring}

The frame structure is analyzed with a two-dimensional beam model. The positions of possible plastic hinges are predefined and approximated as five discrete rigid-perfectly plastic joints, as shown in Figure 2b. Yielding of the frame corners are approximated by one distinct plastic hinge, i.e. Joints 3 and 4, respectively. However, it should be noted that a more accurate approximation can be achieved by modeling the corners as separate substructures. Moreover, several effects are neglected in the analysis, e.g. catenary effects, reduced stiffness due to concrete cracking etc. Nevertheless, the model is suitable for studying different DS techniques and to evaluate the efficiency of the proposed time-stepping method.
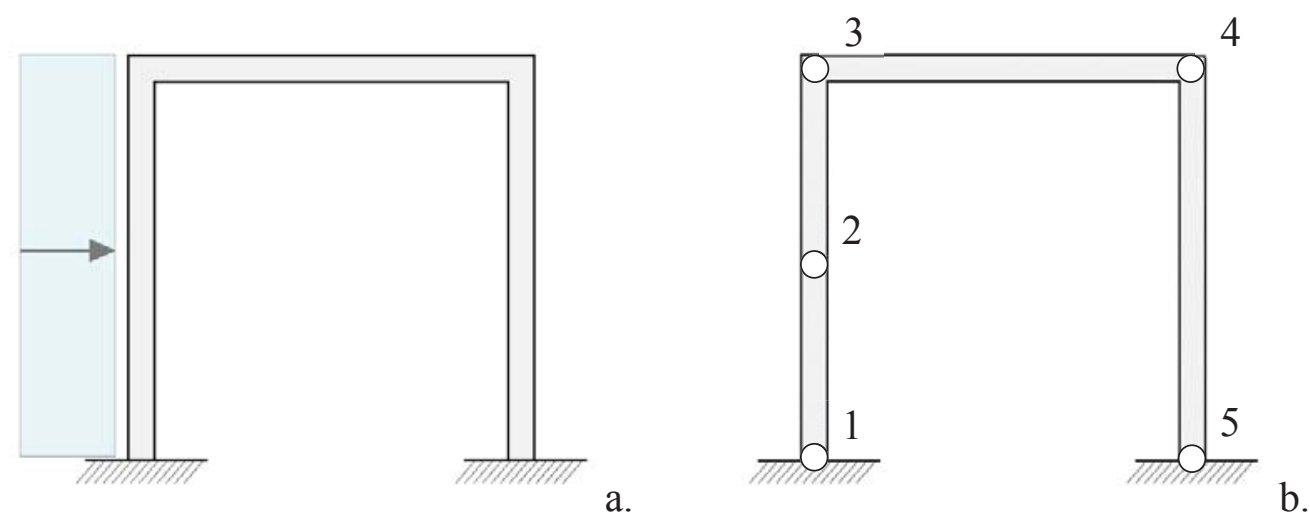

Figure 2: Concrete frame structure subjected to blast loading (a) and locations and labeling of plastic hinges (b). 


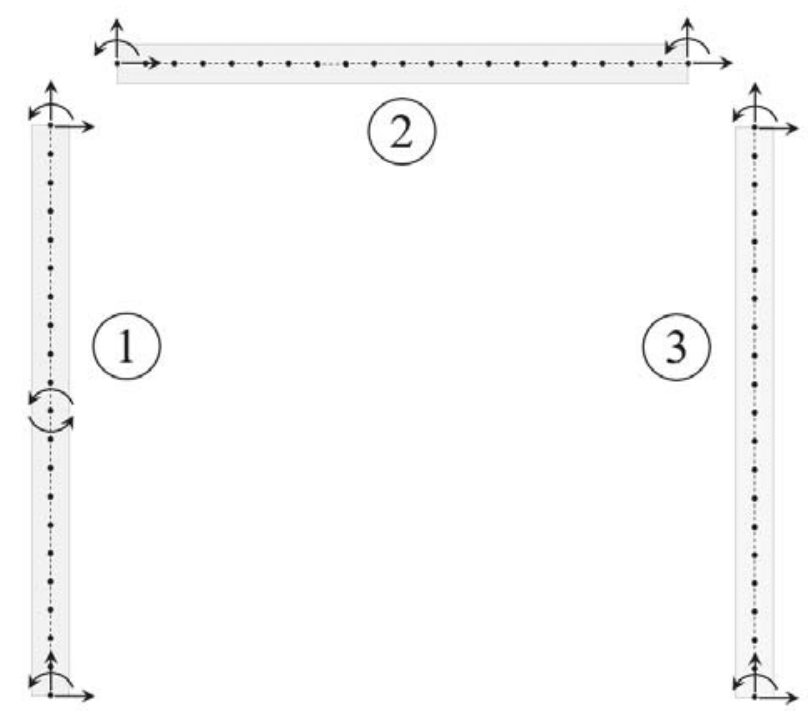

Figure 3: Boundary DOFs and numbering of substructures.

The structure is divided into three substructures, as shown in Figure 3. Each substructure is modeled by 20 Euler-Bernoulli two-node beam elements. Six interface DOFs are retained for Substructures 2 and 3, namely the translational and rotational DOFs at the beam ends. By selecting these boundary DOFs, the fixed-interface normal modes will be the exact mode-shapes of a fixed-fixed beam. Further, eight boundary DOFs are retained for Substructure 1, namely the translational and rotational DOFs at the top and bottom and the rotational DOFs at the joint positioned at midspan. By selecting these boundary DOFs the fixed-interface normal modes will include the exact symmetric modes for a fixed-fixed beam, whereas the antisymmetric modes will be affected by the constrained rotational DOFs at midspan. The fixed-interface normal modes and the constraint modes included in the reduction basis for Substructure 1 is shown in Figures 4 and 5. Note that the antisymmetric modes for a fixed-fixed beam are not excited by a uniformly distributed load. However, since Substructure 1 is not fully supported at the top, some antisymmetric normal modes (modes 2, 5 and 8) are included in the reduction basis.

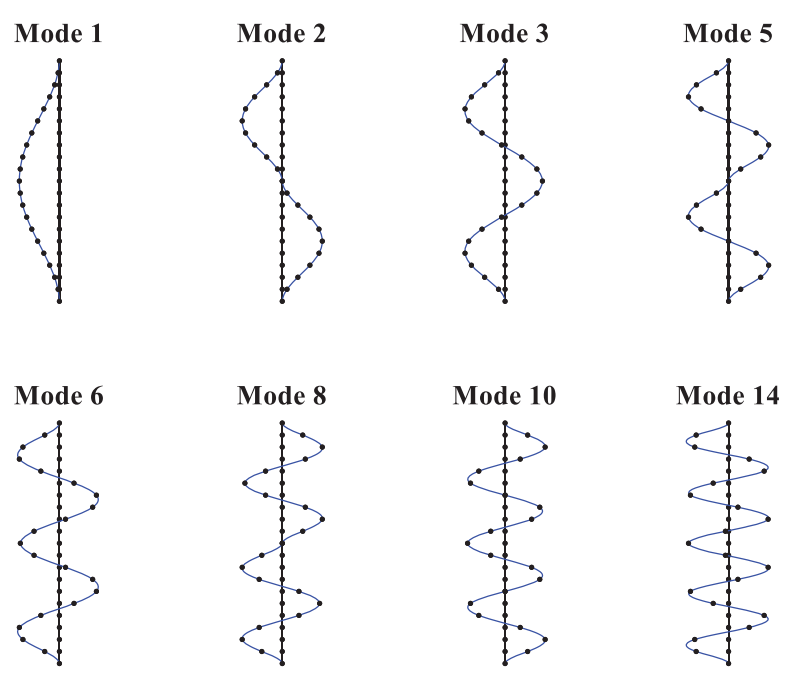

Figure 4: Substructure 1 - fixed-interface normal modes. 


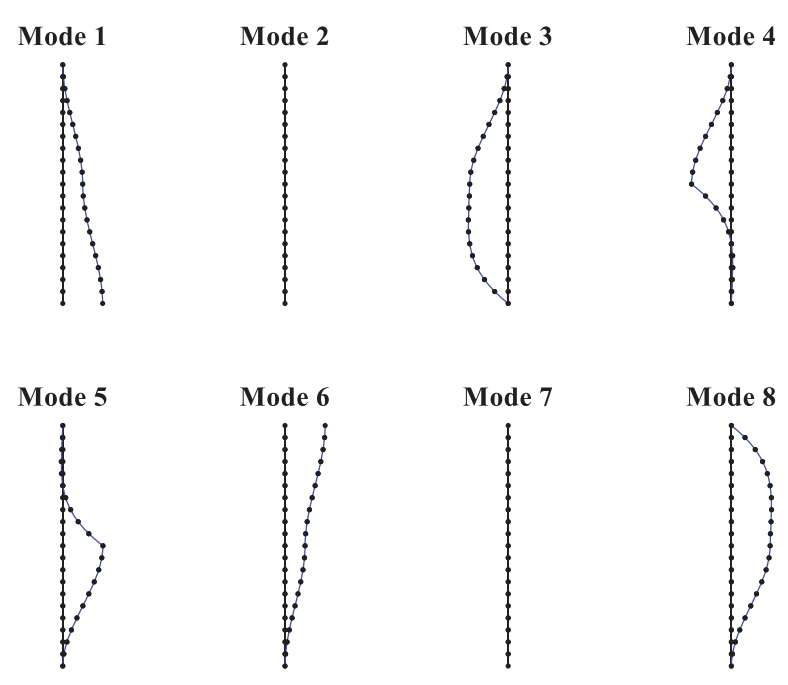

Figure 5: Substructure 1 - constraint modes.

During a dynamic response analysis, the predefined plastic hinges can be either fixed or free, i.e. non-yielding or yielding. A total of five predefined plastic hinges are included in the model, which implies that a response analysis can include up to ten different system configurations. However, to get a rough estimate of the reduced model accuracy two configurations are evaluated-where all joints are either fixed or free, respectively.

The eigenfrequencies for the assembled reduced systems are evaluated by computing the socalled normalized relative frequency difference (NRFD), given by NRFD $=\left|f_{i}^{\text {red }}-f_{i}^{\text {full }}\right| / f_{i}^{f u l l}$, that relate the eigenfrequencies of the reduced system to those of the full system. As previously described, the reduction basis for Substructure 1 includes the fixed-interface normal modes and constraint modes shown in Figures 4 and 5, respectively. Five different reduction bases for Substructures 2 and 3 are evaluated. Using a standard C-B approach, reduction is performed including two or four fixed-interface normal modes. Furthermore, reduction bases including two or four correction modes, as described in Section 3.2, are evaluated. The correction modes are generated based on the constraint modes associated to the rotational boundary DOFs at the beam ends. Hence, two modes are generated by computing a set of first-order correction modes and two additional modes are generated by computing a set of second-order correction modes. In addition, the accuracy of a reduction basis including both four fixed-interface normal modes and two first-order correction modes is evaluated. The analyzed models are summarized in Table 1 .

\begin{tabular}{|c|c|c|c|}
\hline Model name & Normal modes & Correction modes ${ }^{1}$ & No of DOFs ${ }^{2}$ \\
\hline Mode 1-2 & 1,2 & - & 19 \\
\hline Cor. $1^{\text {st }}$ & - & $2 \times 1^{\text {st }}$ & 19 \\
\hline Mode 1-4 & $1,2,3,4$ & - & 23 \\
\hline Cor. $1^{\text {st }}, 2^{\text {nd }}$ & - & $2 \times 1^{\text {st }}+2 \times 2^{\text {nd }}$ & 23 \\
\hline Mode 1-4, Cor. $1^{\text {st }}$ & $1,2,3,4$ & $2 \times 1^{\text {st }}$ & 27 \\
\hline Full model & - & - & 177 \\
\hline
\end{tabular}

${ }^{1}$ Correction modes associated to rotational boundary DOFs.

${ }^{2}$ Total number of DOFs for model with fixed joints.

Table 1: Normal modes and correction modes for Substructures 2 and 3. 
The computed NRFD values are shown in Figures 6 and 7. As shown in the figures, at least, the first seven free and fixed global modes, respectively, are well-described by all the evaluated reduced models; measured as NRFD $<1 \%$. However, to increase the precision for higher order modes, the constraint modes in Substructures 2 and 3 need to be complemented by at least four modes. However, increasing the number of fixed-interface normal modes and/or correction modes in the reduction basis result in additional generalized DOFs in the reduced system, hence, increasing the computational cost. Moreover, the accuracy when using correction modes and normal modes are comparably, as long as the same number of modes are included in the reduction basis.

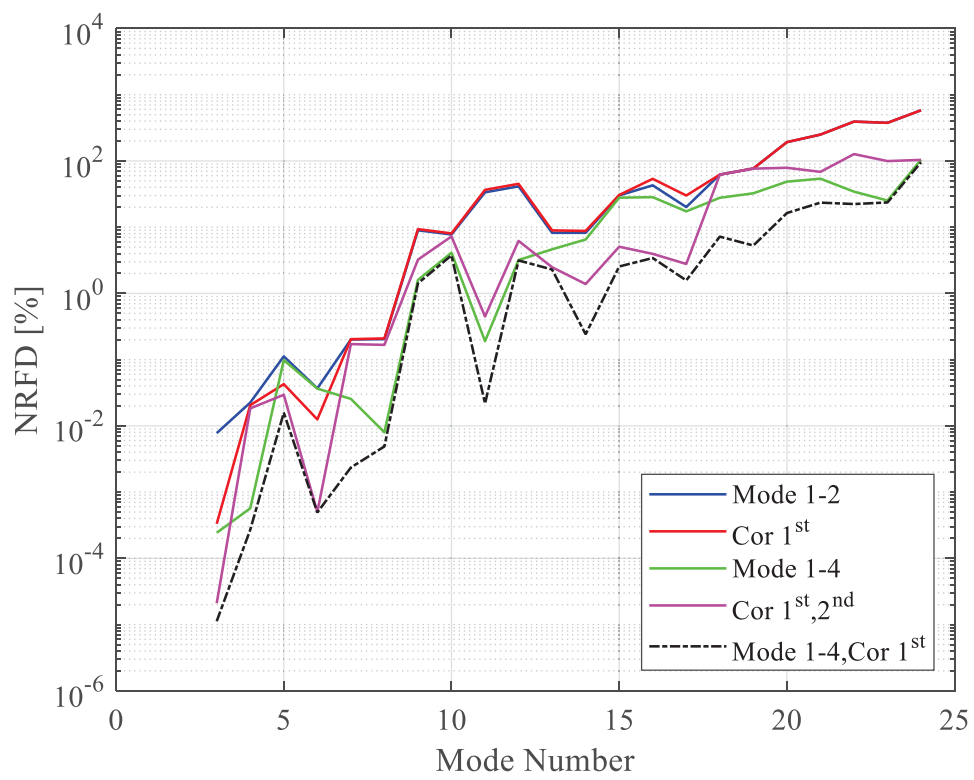

Figure 6: NRFD values for systems with unconstrained rotations at joints.

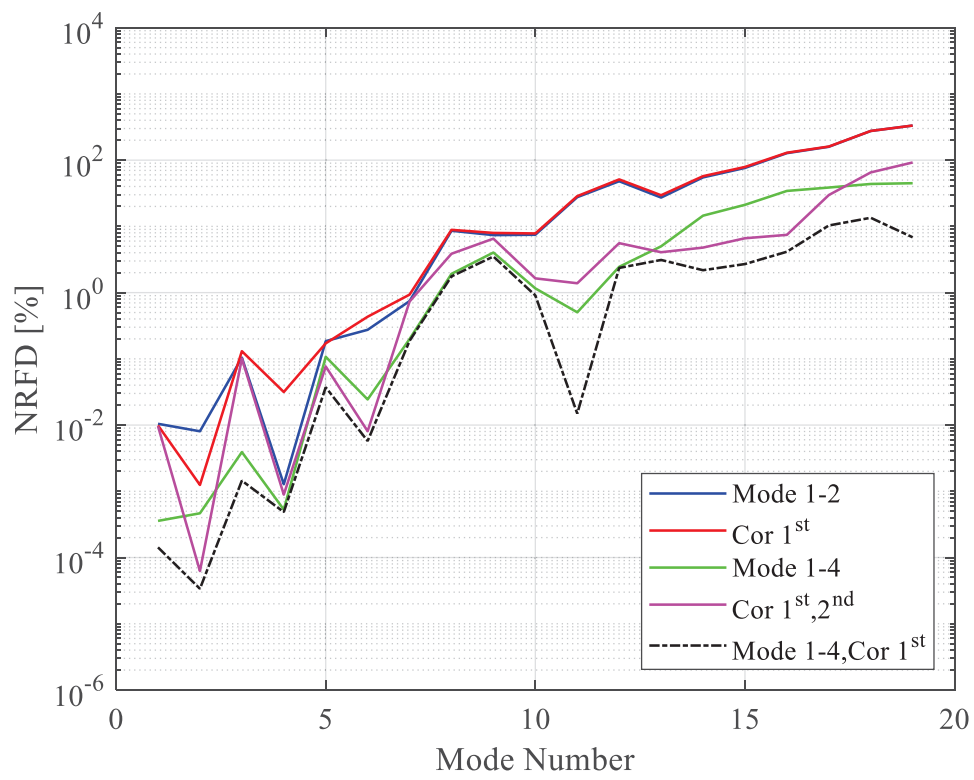

Figure 7: NRFD values for systems with constrained rotations at joints. 

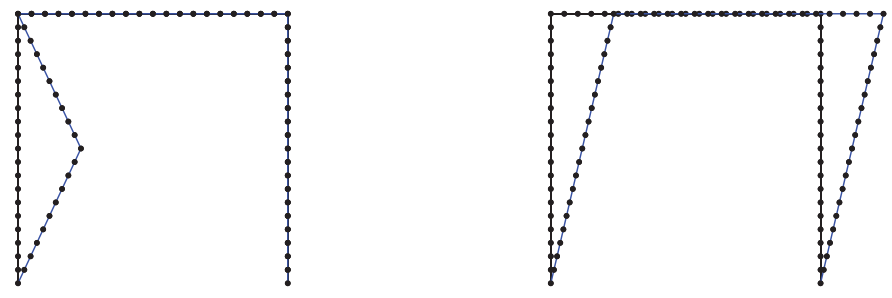

Figure 8: Failure modes spanned by rigid body modes.

Note that the NRFD value is not computed for the first two eigenmodes for the systems with free rotations at the joints. This is due to that the first two modes are rigid body modes with zero frequency. By properly scaling of the rigid body modes, the failure modes, shown in Figure 8 , are obtained.

\subsection{Dynamic response analysis and results}

The dynamic response is computed for the reduced system where the reduction basis for Substructures 2 and 3 includes four normal modes and two first order correction modes. Again, the reduction basis for Substructure 1 includes the normal modes and constraint modes shown in Figures 4 and 5, respectively. The reduced model has a total of 27 DOFs in the initial stage, i.e. when the plastic hinges are fixed, whereas the full model has a total of 177 DOFs. The response is computed using the time-stepping scheme presented in Section 4. Direct time-integration is performed using the Newmark method, with parameters $\beta=1 / 12$ and $\gamma=1 / 2$ that result in a conditionally stable integration scheme with forth order accuracy [19]. The critical time increment is computed as $\mathrm{d} t_{c r i t}=2.45 / \omega_{\max }$. The analysis is performed with a time increment $\mathrm{d} t=\mathrm{d} t_{\text {crit }} / 10$ and the analysis time is set to $150 \mathrm{~ms}$.

Snapshots of the deformed shape during the first $50 \mathrm{~ms}$ is shown in Figure 9. As shown in the figure, the response of the left column during the first, approximately, $10 \mathrm{~ms}$ is similar to that of a fully supported fixed-fixed column due to the inertia of the supporting horizontal beam.

$6 \mathrm{~ms}$

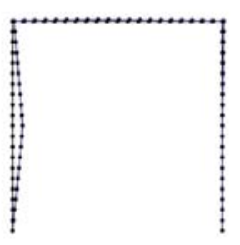

$31 \mathrm{~ms}$

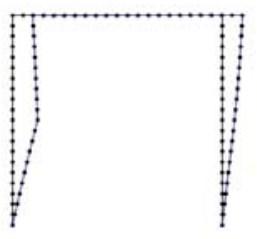

$13 \mathrm{~ms}$

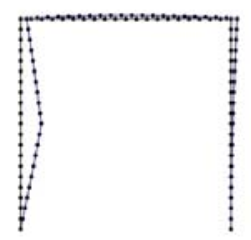

$37 \mathrm{~ms}$

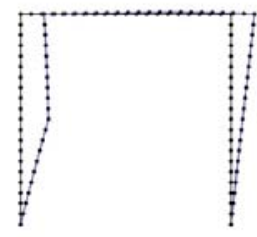

$19 \mathrm{~ms}$

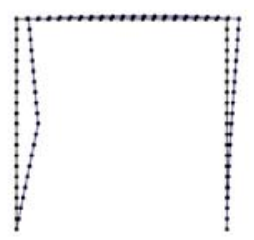

$44 \mathrm{~ms}$

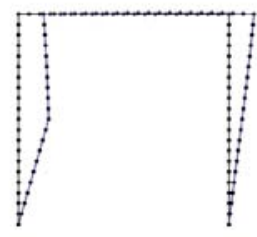

$25 \mathrm{~ms}$

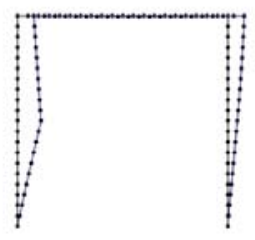

$50 \mathrm{~ms}$

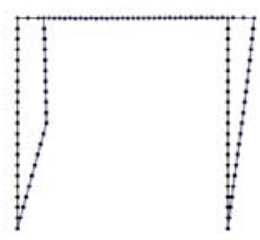

Figure 9: Snapshots of the deformed shape during the first $50 \mathrm{~ms}$. The deformation is scaled by a factor of 15. 


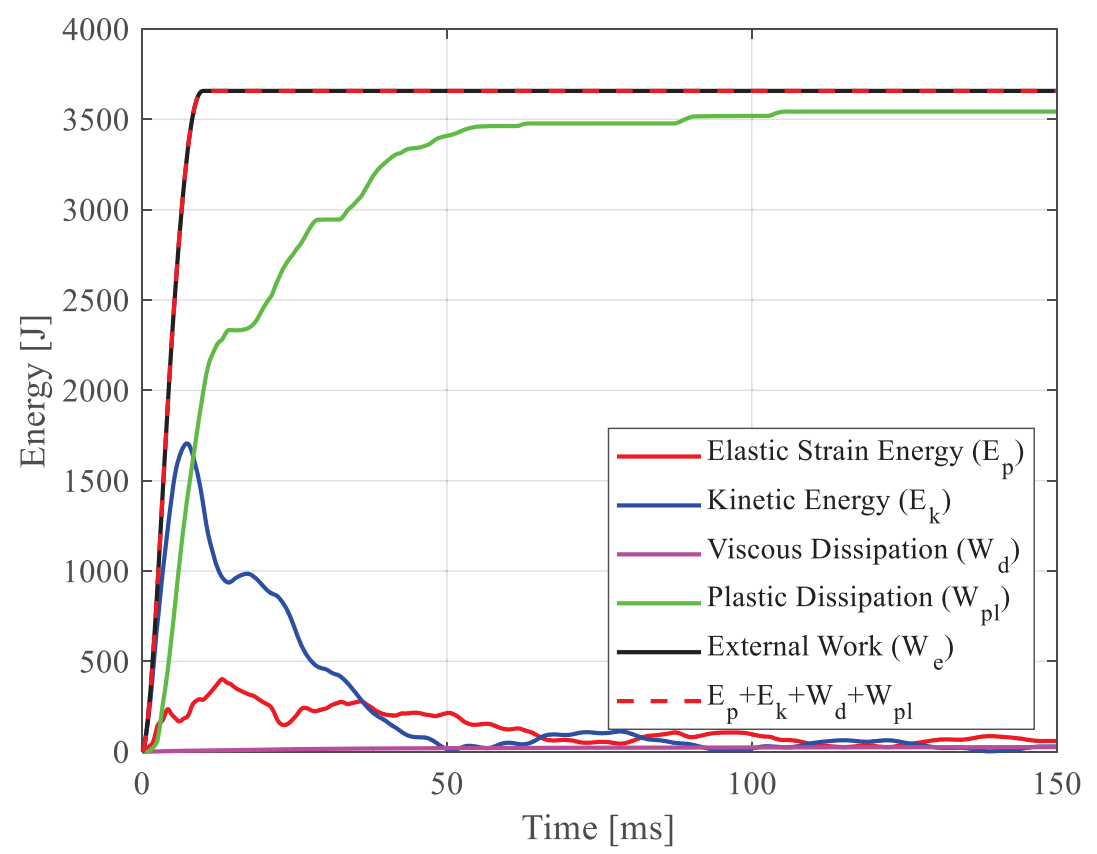

Figure 10: Elastic strain and kinetic energy, plastic and viscous dissipation and external work for the reduced system.

As shown in Figure 10, the energy induced by the external impulse pressure is mainly dissipated by plastic deformations, i.e. by rotation of the predefined plastic hinges. A low Rayleigh beta damping is included in the analysis by setting $\mathbf{C}=10^{-5} \cdot \mathbf{K}$. As shown in the figure, the viscous dissipation is almost zero.

The relative rotation of the plastic hinges is shown in Figure 11. As shown, most of the plastic dissipation is due to plastic rotation in Joints 1 and 2. The rotation computed with the full model is shown in light gray color. As shown in the figure, the response is almost identical to that of the reduced model. Note that the relative rotation of the plastic hinges is an important parameter in a design calculation, which should not exceed the rotation capacity of the crosssection.

The horizontal displacement at Joints 2 and 4 is shown in Figure 12. The displacement is very close to the displacement computed with the full model. The fundamental eigenfrequency for the non-yielding frame structure is $11.7 \mathrm{~Hz}$, corresponding to a period of $1 / 11.7=85 \mathrm{~ms}$. Clearly, the displacement after approximately $50 \mathrm{~ms}$ can essentially be described by the fundamental mode and a plastic displacement. 


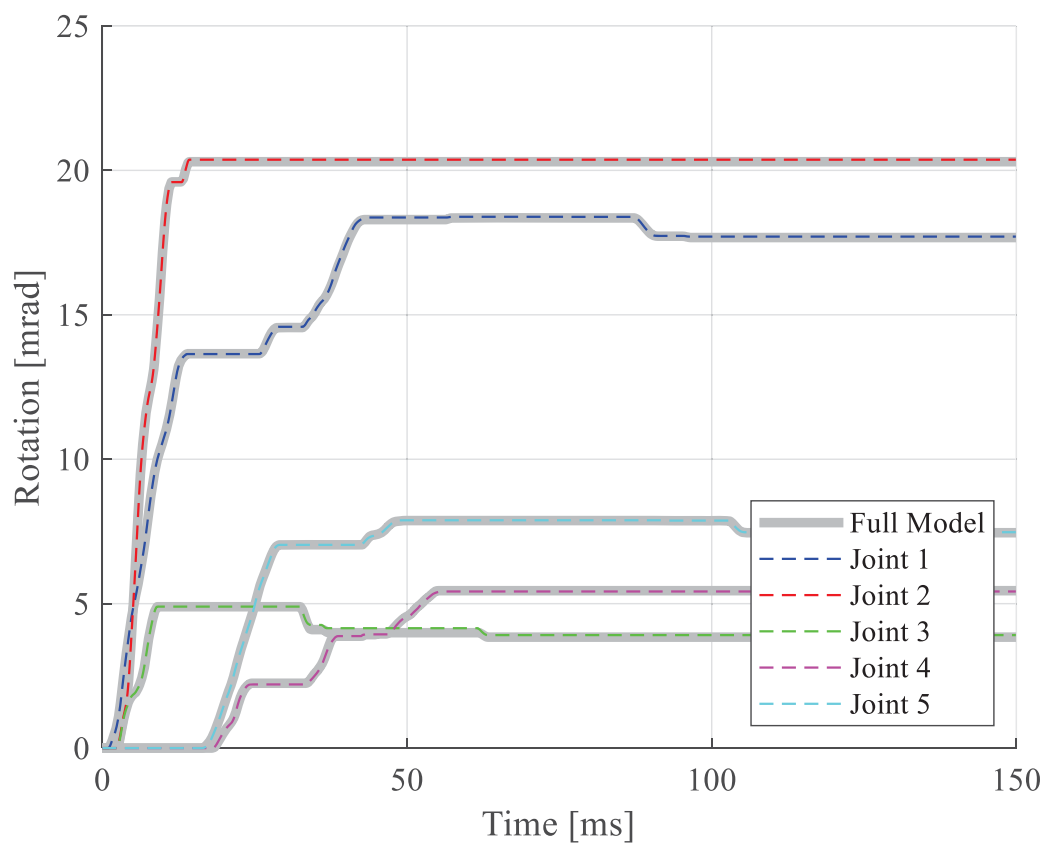

Figure 11: Relative rotations at Joints $1-5$, positioned according to Figure $2 \mathrm{~b}$.

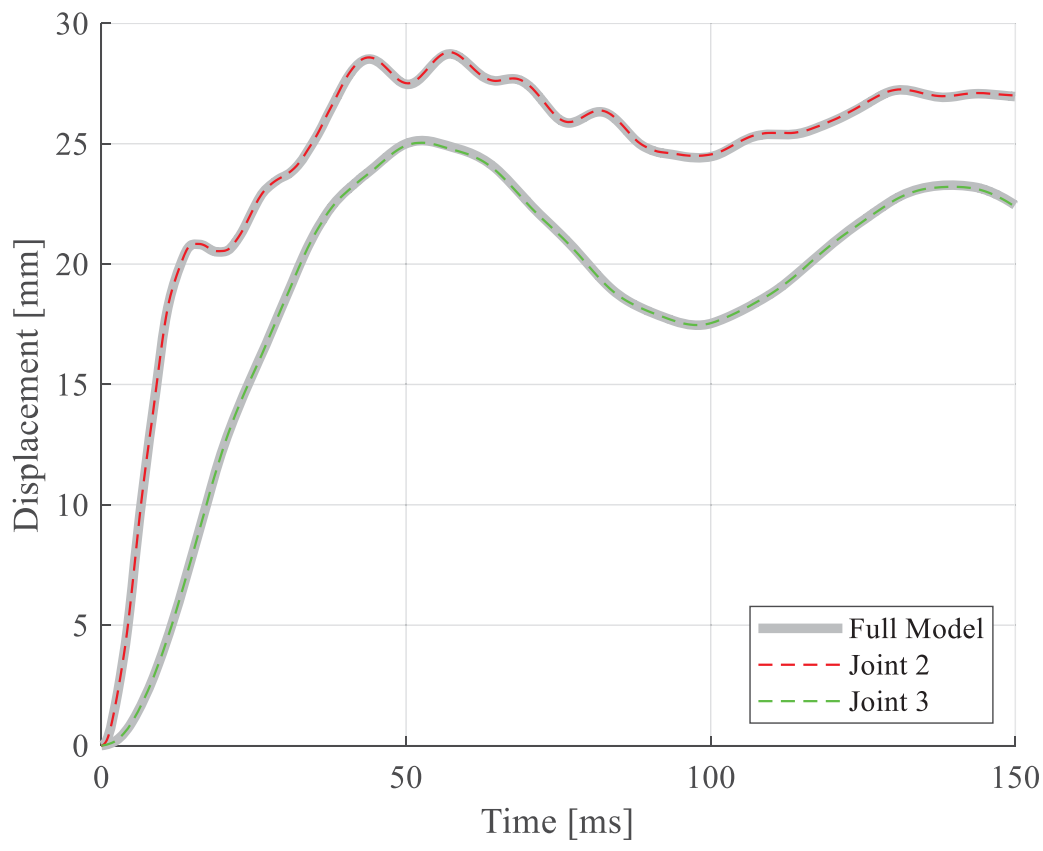

Figure 12: Horizontal displacements at Joints 2 and 3, positioned according to Figure $2 \mathrm{~b}$.

If a suitable reduction basis is applied to Substructure 1, to properly describe the application of the external pressure, the global displacements can be well-described by only including two fixed-interface normal modes (or correction modes) for Substructures 2 and 3, as indicated by Figures 11 and 12. However, a more refined reduction basis is needed to properly describe the reaction and interface forces between the substructures. The influence of the reduction basis on the horizontal interface force in Joint 4 is shown in Figure 13. As shown, the constraint modes need to be complemented by at least four modes to obtain a reasonable accurate response. The 
accuracy for the models reduced by using fixed-interface normal modes and correction modes, respectively, are comparable. Hence, the correction modes are then the preferred choice since the generation of these modes are less computationally expensive (in this simple example, however, the computational cost of generating either normal modes or corrections modes is negligible).

The critical time increment in a conditionally stable direct time integration method is inversely proportional to the highest eigenfrequency. Consequently, a reduction of a system that removes the high frequency content can result in a more computationally efficient analysis. To get an estimation of how much the eigenfrequency is affected by the reduction bases studied herein, the highest eigenfrequency is computed for the initial state of the reduced systems, i.e. when all joints are fixed.

The highest eigenfrequency of the reduced models including two and four fixed-interface normal modes in the reduction bases for Substructure 2 and 3 is $4.2 \mathrm{kHz}$ and $4.3 \mathrm{kHz}$, respectively. The highest eigenfrequency when using two and four correction modes is $4.2 \mathrm{kHz}$ and $4.4 \mathrm{kHz}$, i.e. almost equal to the eigenfrequencies obtained when using normal modes. However, the highest eigenfrequency in the reduced models is in this case most likely governed by Substructure 1 , reduced by a more refined reduction basis.

For the reduced model including four fixed-interface normal modes and two first-order correction modes the highest eigenfrequency is $4.7 \mathrm{kHz}$. In comparison, the highest eigenfrequency of the full model is $74.5 \mathrm{kHz}$, i.e. a factor of $74.5 / 4.7 \approx 16$. Furthermore, the highest eigenfrequency for a reduced system where the rigid-perfectly plastic joints are modeled using penalty elements, with a stiffness 10 times the mean stiffness of the corresponding entries in the unreduced stiffness matrix, is $64.4 \mathrm{kHz}$. Hence, for the model studied herein, the number of time increments can be decreased by a factor $64.4 / 4.7 \approx 14$ by avoiding penalty elements.
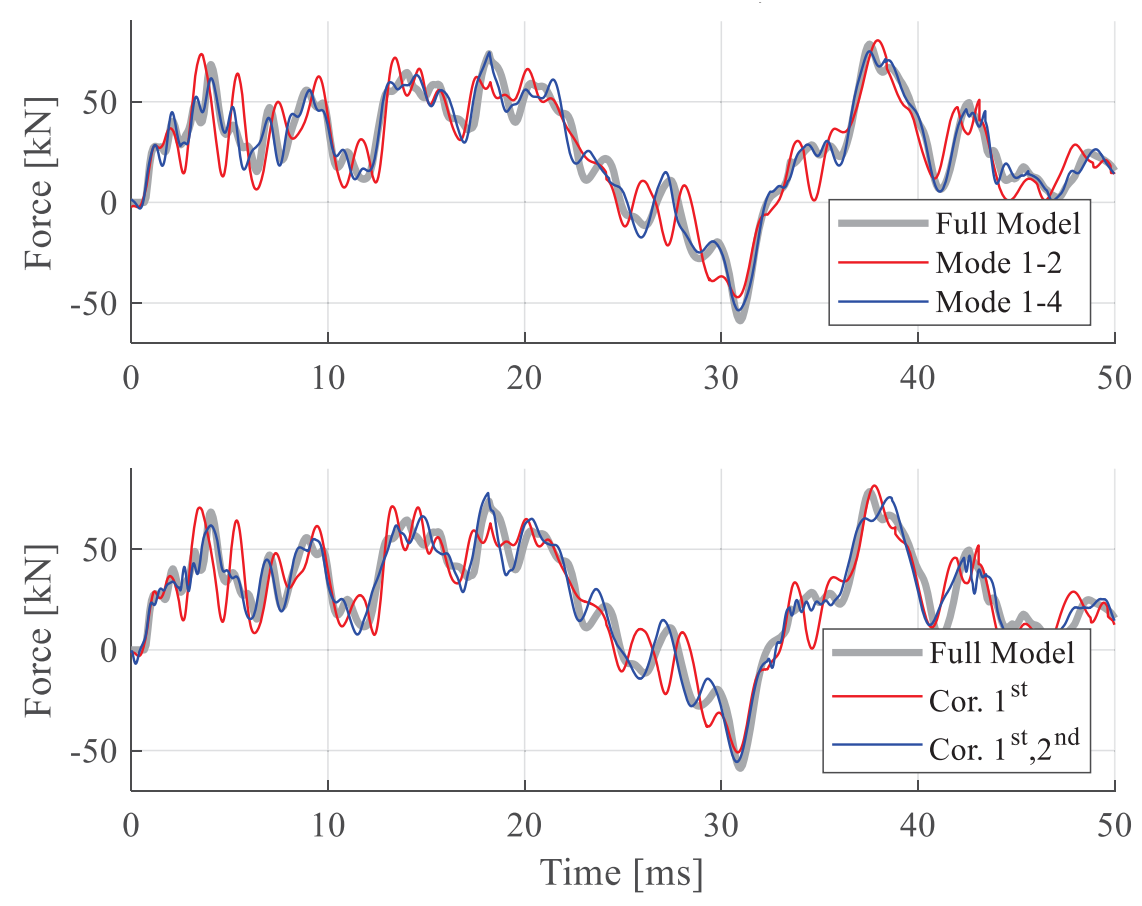

Figure 13: Horizontal interface force at Joint 4. 


\section{CONCLUSIONS}

In the study, strategies are developed to enable time-efficient models for concrete frame structures subjected to blast loading, appropriate for use in a structural design process. Dynamic substructuring is employed to obtain reduced models with localized nonlinearities, such as predefined plastic hinges in a beam-column structure. The parts of the substructures that remains linear elastic are modeled by Ritz-vectors whereas parts with a nonlinear response are retained as physical degrees-of-freedom. Furthermore, a time-stepping scheme is presented that is shown to be suitable for reduced models including local and predefined rigid-plastic behavior.

The proposed methodology is applied and demonstrated in a numerical example of a concrete frame structure. The standard Craig-Bampton method that uses fixed-interface normal modes and constraint modes is evaluated. Furthermore, reduction bases augmented by so-called correction modes are investigated. Various reduction bases are evaluated for the substructures included in the supporting structure, i.e. the substructures that are not subjected to external loading. Two correction modes or two standard fixed-interface normal modes are sufficient to describe the displacement and relative rotations at the plastic hinges. However, to obtain an acceptable accuracy of the interface forces between the substructures, at least four correction modes or four standard fixed-interface modes are needed. For the studied load case, the accuracy when using correction modes and fixed-interface normal modes are comparable.

Moreover, the highest eigenfrequency for the reduced systems, which is related to the critical increment size in conditionally stable direct time integration methods, is evaluated. It is shown that a reduction of the studied model by use of dynamic substructuring can increase the critical time increment by a factor of approximately 16. Furthermore, it is shown that by enforcing a rigid-plastic behavior using the proposed time-stepping scheme, the critical increment size can increase by a factor of approximately 14 compared to a modeling approach where the rigidplastic behavior is enforced using penalty elements.

The present methodology can be extended further to, for example, consider large deformations and failure, e.g. by considering the rotation capacity of the cross-sections. Furthermore, the methods can be applied and evaluated for larger and more complex structures, e.g. threedimensional beam-column structures. Also, a more refined modeling approach for frame corners can be investigated, where the corner itself is treated as a separate substructure.

\section{REFERENCES}

[1] J.M. Biggs, Introduction to Structural Dynamics. McGraw-Hill, New York, 1964.

[2] L. Andersson, P. Persson, P. Austrell, K. Persson, Reduced order modeling for the dynamic analysis of structures with nonlinear interfaces. Proceedings of the $7^{\text {th }}$ ECCOMAS Thematic Conference on Computational Methods in Structural Dynamics and Earthquake Engineering (COMPDYN 2019), Crete, Greece, 24-26 June, 2019.

[3] D. de Klerk, D.J. Rixen, S.N. Voormeeren, General Framework for Dynamic Substructuring: History, Review, and Classification of Techniques, AIAA Journal, 46 (5), 11691181, 2008.

[4] O. Flodén, K. Persson, G. Sandberg, Reduction methods for the dynamic analysis of substructure models of lightweight building structures, Computers and Structures, 138, 4961, 2014. 
[5] Unified Facilities Criteria (UFC), Structures to Resist the Effects of Accidental Explosions, U. S. Army Corps of Engineers, Naval Facilities Engineering Command, Air Force Civil Engineer Support Agency, UFC 3-340-02, December 52008.

[6] R.J. Guyan, Reduction of Stiffness and Mass Matrices, AIAA Journal, 3 (2), 380, 1965.

[7] J. O'Callahan, P. Avitabile, R. Riemer, System Equivalent Reduction Expansion Process (SEREP), Proceedings of the 7th International Modal Analysis Conference, Las Vegas, Nevada, 1989.

[8] R.R. Craig, M.C.C. Bampton, Coupling of Substructures for Dynamic Analysis, AIAA Journal, 6 (7), 1313-1319, 1968.

[9] R.H. MacNeal, A Hybrid Method of Component Mode Synthesis, Computers and Structures, 1 (4), 581-601, 1971.

[10] S. Rubin, Improved Component-Mode Representation for Structural Dynamic Analysis, AIAA Journal, 13 (8), 995-1006, 1975.

[11] R.R. Craig, C.J. Chang, On the Use of Attachment Modes in Substructure Coupling for Dynamic Analysis. Proceedings of the 18th Structures, Structural Dynamics and Material Conference, San Diego, CA, 1977.

[12] D.J. Rixen, A dual Craig-Bampton method for dynamic substructuring, Journal of Computational and Applied Mathematics, 168(1-2), 383-391, 2004.

[13] F.M. Gruber, M. Gille, D.J. Rixen, Time integration of dual Craig-Bampton reduced systems. Proceedings of the $6^{\text {th }}$ ECCOMAS Thematic Conference on Computational Methods in Structural Dynamics and Earthquake Engineering (COMPDYN 2017), Rhodes Island, Greece, 15-17 June, 2017.

[14] R. Craig, A. Hale, Block-Krylov Component Synthesis Method for Structural Model Reduction. Journal of Guidance, Control and dynamics, American Institute of Aeronautics and Astronautics, 11(6), 562-570, 1988.

[15] D.J. Rixen, High Order Static Correction Modes for Component Mode Synthesis. Proceedings of the fifth World Congress on Computational Mechanics, Vienna, Austria, 712 July, 2002.

[16] R.R. Jr. Craig, A.J. Kurdila, Fundamentals of Structural Dynamics, $2^{\text {nd }}$ Edition, John Wiley \& Sons, New Jersey, 2006.

[17] K. J. Bathe, Finite element procedures. Prentice-Hall, 1996.

[18] C. Farhat, L. Crivelli, M. Géradin, Implicit time integration of a class of constrained hybrid formulations-Part I: Spectral stability theory, Computer Methods in Applied Mechanics and Engineering, 125 (1-4), 71-107, 1995.

[19] M. Geradin, D.J. Rixen, Mechanical Vibrations: Theory and Applications to Structural Dynamics, Third Edition. Wiley, New York, 2014. 